\title{
Combined Visco-Trab operation: A dual filtration pathway for management of advanced glaucoma-midterm results
}

\author{
Tarek M. Eid (1D) Ezz El-Din M. Ibrahim • Ahmad Zaid
}

Received: 27 June 2020/ Accepted: 8 January 2021 / Published online: 6 February 2021

(C) This is a U.S. government work and not under copyright protection in the U.S.; foreign copyright protection may apply 2021

\begin{abstract}
Purpose To study midterm efficacy and safety of combined Visco-Trab operation for management of advanced glaucoma.

Methods 168 eyes of 148 patients with advanced glaucoma had Visco-Trab operation (a merge of both viscocanalostomy and trabeculectomy operations). Mean follow-up was $29.1 \pm 22.2$ months. Criteria of success were intraocular pressure (IOP) of $14 \mathrm{mmHg}$ or less with or without glaucoma medications, with no
\end{abstract}

This study was presented as paper in the ASCRS ASOA Annual Meeting, San Diego, California, May 3-7, 2019

Supplementary Information The online version contains supplementary material available at (https://doi.org/10.1007/ s10792-021-01698-0).

T. M. Eid ( $\square)$

Ophthalmology Department, Faculty of Medicine, Tanta

University, Tanta, Egypt

e-mail: tarekmeid@hotmail.com

T. M. Eid

Glaucoma and Cataract Unit, Magrabi Hospitals and

Centers, Cairo, Egypt

T. M. Eid

EyeCity Center, New Cairo, Egypt

E. E.-D. M. Ibrahim

Glaucoma and Cataract Unit, Magrabi Hospitals and

Centers, Riyadh, Saudi Arabia devastating complications, loss of light perception, or additional glaucoma surgery.

Results IOP, number of glaucoma drops, and visual field mean deviation were significantly reduced $(11.9 \pm 5.6 \mathrm{mmHg}, 0.7 \pm 1.2$, and $14.2 \pm 6.3 \mathrm{~dB}$, compared to preoperative values of $24.4 \pm 9.9 \mathrm{mmHg}, 2.8 \pm 1.4$, and $17.3 \pm 6.3 \mathrm{~dB}$, respectively). Success was reported in 136 of 168 eyes $(81 \%)$ without (100 eyes, $59.5 \%)$ or with (36 eyes, $21.5 \%$ ) glaucoma medications. A functioning bleb was seen in 2/3rd of eyes; diffuse (59 eyes, $35 \%$ ) and thin ischemic (54 eyes, 32\%). Predictors for failure to achieve the target IOP included previous ocular $(p=0.01)$ or glaucoma $(p=0.04)$ surgery, number of preoperative glaucoma medications $(p=0.029)$, and severity of glaucoma $(p=0.058)$.

A. Zaid

Glaucoma and Cataract Unit, Magrabi Hospitals and Centers, Mascat, Oman 
Conclusion Combined Visco-Trab operation proved safe and effective, on midterm follow-up, in reducing IOP to the proposed target level in eyes with severe glaucoma via enhancing internal and external filtration.

Keywords Advanced glaucoma - Visco-trab operation $\cdot$ Dual filtration $\cdot$ Midterm results

\section{Introduction}

Patients with advanced glaucoma (near total cupping of the optic disc and advanced visual field defect encroaching on fixation) are high-risk surgical candidates [1]. Complications of current surgical interventions, namely subscleral trabeculectomy with mitomycin $\mathrm{C}$ application (MMC-Trab), include high incidence of hypotony-related complications after the filtering operation or after suture cutting (flat anterior chamber, choroidal effusion or hemorrhage etc.) or postoperative pressure rise due to tight flap closure. This turbulence of postoperative IOP may endanger the compromised optic nerve and the residual vision[2].

Visco-Trab operation constitutes combining viscocanalostomy and trabeculectomy with Mitomycin C in a single technique in order to increase both internal and external flow of aqueous postoperatively [3]. Surgical technique included lamellar and deep scleral flap dissection, deroofing and viscodilation of Schlemm's canal (SC), penetrating trabeculectomy, peripheral iridectomy, and tight flap closure. The early postoperative IOP reduction after Visco-Trab was mostly attributed to improved internal flow mechanisms, while external filtration is minimized by tight scleral flap closure. Subsequent scleral flap suture cutting, when needed, allows subconjunctival drainage of aqueous. Persistent IOP lowering is attributed partly to improved conventional outflow pathways and partly to external filtration. The controlled hypotony produced by enhancing internal filtration and limiting external filtration plays a major role in reducing IOP to the target level during the early postoperative period without pressure spikes or severe complications related to excessive filtration [3]. In a comparative study of the contralateral eye, comparing combined Visco-Trab operation to MMC-Trab proved a similar efficacy over a longer follow-up period with reduced postoperative complications [4].

In this study we evaluated the midterm efficacy and safety of Visco-Trab operation in terms of controlling IOP to a low and stable level with less eventful postoperative course.

\section{Patients and methods}

A retrospective analysis of collected data for previous prospective studies was conducted. 168 eyes of 148 patients with advanced to end-stage glaucoma surgically treated with Visco-Trab operation in the Glaucoma and Cataract Unit at Magrabi Eye and Ear Center, Jeddah, Saudi Arabia, from 2006 till December 2013 were included in the analysis. A local institutional review board was obtained which meets the tenets of the Declaration of Helsinki, and an informed consent was signed by every patient. Advanced glaucoma in this study was defined as near total cupping of the optic disc, severe visual field defect encroaching on central 5 degrees of fixation in at least one quadrant or tubular field defect [1]. In some patients, reliable visual field cannot be obtained because of poor vision, dense cataract, or unreliability of the test. For these patients, glaucoma severity was judged by degree of damage of optic disc, IOP, and patient's compliance. Surgical treatment for these patients was indicated when there was lack of proper control of IOP to the required target level with maximally tolerated medical therapy. The risk-benefit ratio of surgical management was explained to the patient, and a consent form was obtained. Patients with visually significant cataract had separate-site phacoemulsification combined with the glaucoma procedure.

\section{Surgical technique (Fig. 1)}

The procedure was performed under topical or peribulbar anesthesia without application of Honan bulb. General anesthesia was given in young or uncooperative or sometimes in single-eyed patients. The globe is rolled down with superior corneal traction suture with $7 / 0$ silk. The filtering site is located slightly nasal or temporal to the 12 o'clock position. Details of the procedure are explained in previous studies [3, 4]. 


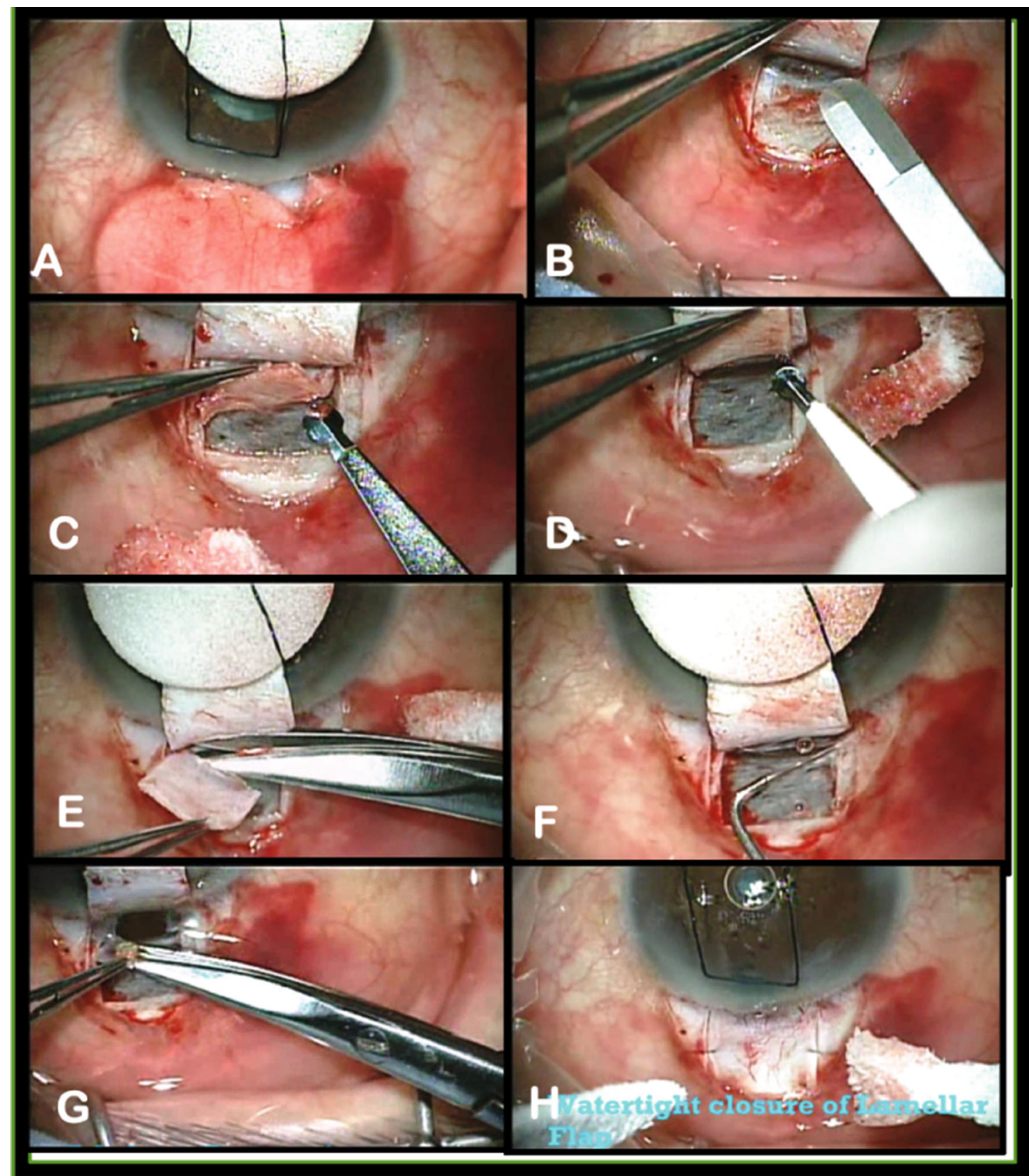

Fig. 1 Steps of combined Visco-Trab operation for advanced glaucoma. a Fornix-based conjunctival flap and subconjunctival Mitomycin C $0.3 \mathrm{mg}$ for $3 \mathrm{~min}$. b A $4 \times 4 \mathrm{~mm}$ lamellar scleral flap (1/3-1/2 of thickness) extending $1 \mathrm{~mm}$ in clear cornea. c Deep scleral flap dissection $0.5 \mathrm{~mm}$ inside edge, leaving thin scleral over choroid d Exposure \& deroofing of Schlemm's

After preparing the site of surgery and Mitomycin C application, a $4 \times 4 \mathrm{~mm}$ rectangular lamellar sclera flap was outlined and dissected anteriorly to within canal (No Descemet's window exposure). e Excision of deep scleral flap creating a scleral lake. f Dilation of SC with sodium hyaluronate. $g$ Penetrating trabeculectomy and peripheral iridectomy. h Water tight closure of lamellar scleral flap and conjunctival flap

$1 \mathrm{~mm}$ of clear cornea. A second deeper rectangular flap was outlined $0.5 \mathrm{~mm}$ inside the border of the first and dissected using a special dissecting sclera pocket 
knife (Grieshaber, Switzerland), leaving only a thin translucent layer overlying the choroid. Forward dissection in this plane continues until Schlemm's canal (SC) is identified and deroofed. Dissection continues through the lumen and extends forward until it exceeds Schwalbe's line and Descemet's membrane is seen. No blunt dissection of Descemet's window is performed as in routine viscocanalostomy. A specially designed viscocanalostomy cannula (Grieshaber, Switzerland), with an outer diameter of $165 \mu \mathrm{m}$ was introduced into the ostia of SC, right and left, to inject sodium hyaluronate $1 \%$ (ProVisc, Alcon) into the canal. The cannula is introduced inside the canal for a short distance, and a small amount of viscoelastic is injected and repeated few times on each side. The trabeculo-Descemet's membrane is incised right at the junction with the anterior border of the floor of SC, and a penetrating deep sclero-corneal block was excised for approximately $1.5 \mathrm{~mm}$ in length and $2 \mathrm{~mm}$ in width. A wide peripheral iridectomy is performed, and the lamellar sclera flap is closed watertight by $410 / 0$ Nylon sutures, 2 at the distal corners and 2 on the vertical limbs. The AC is reformed, and the conjunctiva is closed with 2 corners and 2 transverse-mattress 10/0 Nylon sutures.

When the glaucoma procedure is combined with phacoemulsification, once Schlemm's canal is deroofed, the surgeon switches his position to the temporal side to do the cataract operation through a clear-cornea incision. At the end of the phaco procedure, the $\mathrm{AC}$ is reformed and the corneal wound is secured by one 10/0 Nylon suture and the surgeon reverts back to upper position to complete the filtering operation.

Postoperatively patients used antibiotic-corticosteroid eye drops every $2 \mathrm{~h}$ for one week and then tapered gradually over 5 weeks. Cycloplegic-mydriatic eye drops were used for one week and may be continued if there were signs of early inflammation, shallow AC, hypotony, or anticipated risk of aqueous misdirection. Patients were examined at day 1 and 3 then at 1,2,4, and 8 weeks and after that periodically every 3 months. If the pressure was elevated, digital massage, focal compression, and LSL, needling with flap lift, or bleb revision with subconjunctival MMC injection were performed according to the IOP level and degree of filtration.

A minimal of 3 months follow-up was required to be included in the analysis. Criteria of Success were
IOP of $14 \mathrm{mmHg}$ or less at the last follow-up, with no devastating postoperative complications or loss of vision or additional glaucoma surgery.

Descriptive statistics of numeric (mean \pm standard deviation) and categorical (count and percentage) variables for patients' eyes, and glaucoma characteristics, operative data, and postoperative course were listed and tabulated. Paired t-test was used to compare IOP, number of glaucoma medications, visual field mean deviations, horizontal cup-disc ratio, and logMar visual acuity preoperatively and at the last follow-up evaluation. Kaplan-Meier survival analysis of probability of failure of Visco-Trab operation to achieve an IOP of $14 \mathrm{mmHg}$ or less was plotted over the follow-up period. Predictors of failure to achieve the target pressure were studied using logistic regression analysis with a significance level of $p \leq 0.05$.

\section{Results}

One hundred ninety-seven eyes were operated with Visco-Trab operation throughout the study period, 25 of them had less than 3 months of follow-up in their records and did not match the inclusion criteria. Additional 4 eyes were converted to classic MMCTrab and were excluded from the analysis.

Table 1 lists demographic data, characteristics of the study eye and type and severity of glaucoma. Seventy percent of the study patients were males (103 patients) and almost $60 \%$ of them (87 patients) were above 50 years of age. More than half of the eyes had primary open-angle glaucoma and 20\% (34 eyes) had primary angle closure glaucoma. Three quarters of the eyes received previous glaucoma treatment, and twothirds of them had a preoperative IOP of $25 \mathrm{mmHg}$ or less. Table 2 describes operative data, intraoperative complications, early postoperative course, postoperative complications and additional surgical interventions. Self-absorbing hyphema was the most common early postoperative complications (21 eyes, 12.5\%) followed by shallow to flat anterior chamber (19 eyes, $11.4 \% ; 3$ eyes required added maneuvers to reform the anterior chamber). Three eyes (1.8\%) suffered from postoperative hypotony with choroidal effusion, two of them required choroidal tap to drain the fluid. Scleral flap lift with a 27 -gauge needle on the slitlamp (14 eyes, $8.3 \%$ ) or bleb revision with additional subconjunctival mitomycin $\mathrm{C}$ injection (11 eyes, 
Table 1 Patients' and eyes' characteristics $(n=168)$

\begin{tabular}{|c|c|c|c|}
\hline Variable & & Frequency & Percentage \\
\hline $\operatorname{Sex}(n=148)$ & Male & 103 & 69.6 \\
\hline Age group $(n=148)$ & $>50$ years & 87 & 58.8 \\
\hline Study eye & Right eye & 81 & 48.2 \\
\hline Previous glaucoma treatment & & 125 & 74.4 \\
\hline Previous glaucoma surgery (MMC-Trab) & & 27 & 16.1 \\
\hline \multirow[t]{3}{*}{ Preoperative visual acuity } & $>20 / 40$ & 93 & 55.4 \\
\hline & 20/40-20/200 & 53 & 31.5 \\
\hline & $</=20 / 400$ & 22 & 13.1 \\
\hline \multirow[t]{2}{*}{ Preoperative IOP (mmHg) } & $</=25$ & 111 & 66.1 \\
\hline & $>25$ & 57 & 33.9 \\
\hline \multirow[t]{3}{*}{ Lens status } & Clear & 116 & 69.0 \\
\hline & Cataract & 24 & 14.3 \\
\hline & Pseudophakic & 28 & 16.7 \\
\hline \multirow[t]{7}{*}{ Preoperative visual field changes } & Double arcuate scotoma & 6 & 3.6 \\
\hline & Hemifield defect & 6 & 3.6 \\
\hline & Advanced defect not affecting fixation & 17 & 10.1 \\
\hline & Advanced defect splitting fixation & 64 & 38.1 \\
\hline & Tubular field defect & 21 & 12.5 \\
\hline & Total field loss & 12 & 7.2 \\
\hline & Not done & 42 & 25.0 \\
\hline \multirow[t]{10}{*}{ Glaucoma diagnosis } & Primary open-angle & 89 & 53.0 \\
\hline & Low-tension & 4 & 2.4 \\
\hline & Primary angle closure & 34 & 20.3 \\
\hline & Congenital & 14 & 8.3 \\
\hline & Juvenile & 8 & 4.8 \\
\hline & Angle recession & 8 & 4.8 \\
\hline & Pseudoexfoliative & 5 & 3.0 \\
\hline & Pigmentary & 2 & 1.2 \\
\hline & Steroid-induced & 3 & 1.8 \\
\hline & Uveitic & 1 & 0.6 \\
\hline \multirow[t]{2}{*}{ Glaucoma severity } & Advanced & 121 & 72.0 \\
\hline & End-stage & 47 & 28.0 \\
\hline
\end{tabular}

$6.5 \%)$ were done in eyes after failure of laser suture lysis to augment external filtration.

Mean follow-up was $29.1 \pm 22.2$ months (range 3-86). Mean pre- and postoperative IOP, number of glaucoma medications, visual acuity, and visual field mean deviation are demonstrated in Table 3. Mean IOP and number of glaucoma medications were reduced significantly at the last follow-up evaluation in comparison with their preoperative levels (11.9 versus $24.4 \mathrm{mmHg}$ and 0.7 versus 2.8 , respectively, $p<0.001)$. On the other hand, the mean values of cupdisc ratio, visual field mean deviation, and logMar of visual acuity showed stable course over the follow-up length without any significant change from preoperative values.

Table 4 presents the number and percentage of eyes that achieved an IOP of $21 \mathrm{mmHg}$ or less with or without antiglaucoma medications, as well as the number and percentage of eyes that did or did not achieve the target pressure $(\leq 14 \mathrm{mmHg})$ at last 
Table 2 Operative data and postoperative course of study eyes after Visco-Trab operation

\begin{tabular}{|c|c|c|c|}
\hline Variable & & Frequency & Percentage \\
\hline \multirow[t]{3}{*}{ Anesthesia } & Topical & 82 & 48.8 \\
\hline & Peribulbar & 68 & 40.5 \\
\hline & General & 18 & 10.7 \\
\hline \multicolumn{4}{|l|}{ Intraoperative complications } \\
\hline & Vitreous loss from sclerostomy & 2 & 1.2 \\
\hline & $\begin{array}{l}\text { Descemet membrane detachment during } \\
\text { viscodilation }\end{array}$ & 3 & 1.8 \\
\hline & Choroidal exposure during deep flap dissection & 4 & 2.4 \\
\hline \multicolumn{4}{|l|}{$\begin{array}{l}\text { IOP measurement within the first postoperative } \\
\text { week }\end{array}$} \\
\hline & $</=10 \mathrm{mmHg}$ & 133 & 79.2 \\
\hline & $>10 \mathrm{mmHg}$ & 35 & 20.8 \\
\hline \multirow[t]{7}{*}{ Early postoperative complications } & & 38 & 22.6 \\
\hline & Hyphema & 21 & 12.5 \\
\hline & Shallow anterior chamber & 16 & 9.6 \\
\hline & Flat anterior chamber & 3 & 1.8 \\
\hline & Leaking conjunctival hole & 2 & 1.2 \\
\hline & Choroidal effusion & 3 & 1.8 \\
\hline & Suprachoroidal hemorrhage & 1 & 0.6 \\
\hline \multicolumn{4}{|l|}{ Postoperative laser suture lysis } \\
\hline & Within one week & 4 & 2.4 \\
\hline & Between 1 and 4 weeks & 36 & 21.4 \\
\hline & Between 1 and 3 months & 5 & 3.0 \\
\hline \multicolumn{4}{|l|}{ Added postoperative surgical intervention } \\
\hline & Anterior chamber reformation & 3 & 1.8 \\
\hline & Repair of conjunctival hole & 2 & 1.2 \\
\hline & Scleral flap lift on slitlamp & 14 & 8.3 \\
\hline & Bleb revision and subconj 5-MMC & 11 & 6.5 \\
\hline & Choroidal drainage & 2 & 1.2 \\
\hline & Repair of Descemet membrane detachment & 1 & 0.6 \\
\hline Cataract extraction during postoperative course & & 12 & 7.1 \\
\hline
\end{tabular}

follow-up visit. Eighty one percent of eyes (136 of 168 eyes) achieved a target IOP of $14 \mathrm{mmHg}$ or less without (100 eyes, $59.5 \%)$ or with glaucoma drops (36 eyes, $21.4 \%$ ). This percentage of success (both complete and qualified) increased to $95.3 \%$ when a pressure of $21 \mathrm{mmHg}$ or less was used as a cutoff value. The recorded appearance of the filtering bleb at last visit examination was counted and is presented in Table 4. Almost two third of the eyes had a functioning bleb either diffuse (59 eyes, $35 \%$ ) or thin ischemic (54 eyes, $32 \%$ ) blebs. Cumulative probability of failure of
Visco-Trab operation to achieve an IOP of $14 \mathrm{mmHg}$ or less over the extended follow-up period was shown in the Kaplan-Meier analysis chart (Fig. 2).

Predictors for failure to achieve the target IOP of $14 \mathrm{mmHg}$ or less at last follow-up evaluation (by logistic regression analysis) included previous ocular ( $p=0.01)$ or glaucoma $(p=0.04)$ surgery, number of preoperative antiglaucoma medications $(p=0.029)$, severity of glaucoma $(p=0.058)$. Nonsignificant predictors in the multivariate model included old age 
Table 3 Mean, standard deviation, minimum, and maximum values of numeric variables preoperatively and at the last follow-up in the study

\begin{tabular}{|c|c|c|c|c|c|c|}
\hline Variable & & Mean & SD & Minimum & Maximum & $P$ value \\
\hline Age (years) & & 51.1 & 18.9 & 4 & 85 & \\
\hline Length of follow-up (months) & & 29.1 & 22.2 & 3 & 86 & \\
\hline \multirow[t]{2}{*}{ IOP $(\mathrm{mmHg})$} & Preoperative & 24.4 & 9.9 & 10 & 55 & \multirow[t]{2}{*}{$<0.001^{*}$} \\
\hline & At last follow-up & 11.9 & 5.6 & 1 & 31 & \\
\hline \multirow[t]{2}{*}{ Number of glaucoma drops } & Before surgery & 2.8 & 1.4 & 0 & 5 & \multirow[t]{2}{*}{$<0.001^{*}$} \\
\hline & At last follow-up & 0.7 & 1.2 & 0 & 4 & \\
\hline \multirow[t]{2}{*}{ Cup/disc ratio (horizontal) } & Before surgery & 0.9 & 0.1 & 0.5 & 1.0 & \multirow[t]{2}{*}{0.9} \\
\hline & At last follow-up & 0.9 & 0.4 & 0 & 1.0 & \\
\hline \multirow[t]{2}{*}{ Visual field mean deviation $(\mathrm{dB})$} & Preoperative & 17.3 & 6.7 & 6.2 & 32.4 & \multirow[t]{2}{*}{0.2} \\
\hline & At last follow-up & 14.2 & 6.3 & 4.5 & 24 & \\
\hline \multirow[t]{2}{*}{ LogMar of visual acuity } & Preoperative & 0.5 & 0.3 & 0 & 1.0 & \multirow[t]{2}{*}{0.9} \\
\hline & At last follow-up & 0.5 & 0.3 & 0 & 1.0 & \\
\hline
\end{tabular}

Table 4 Complete and qualified success of achieving a postoperative IOP of $21 \mathrm{mmHg}$ and $14 \mathrm{mmHg}$ and the appearance of the associated filtering bleb at last follow-up evaluation

\begin{tabular}{|c|c|c|c|}
\hline \multicolumn{2}{|l|}{ Variable } & \multirow[t]{2}{*}{ Frequency } & \multirow[t]{2}{*}{ Percentage } \\
\hline Filtering & & & \\
\hline & Flat bleb & 23 & 13.7 \\
\hline & Localized bleb & 28 & 16.6 \\
\hline & Diffuse bleb & 59 & 35.1 \\
\hline & Thin ischemic bleb & 54 & 32.1 \\
\hline & Cystic bleb & 4 & 2.4 \\
\hline \multicolumn{4}{|c|}{ IOP $<21 \mathrm{mmHg}$ at last follow-up } \\
\hline & Without antiglaucoma drops & 130 & 77.4 \\
\hline & With antiglaucoma drops & 30 & 17.9 \\
\hline & IOP higher than $21 \mathrm{mmHg}$ & 8 & 4.8 \\
\hline \multicolumn{4}{|c|}{ IOP $</=14 \mathrm{mmHg}$ at last follow-up } \\
\hline & Without antiglaucoma drops & 100 & 59.5 \\
\hline & With antiglaucoma drops & 36 & 21.4 \\
\hline & IOP higher than $14 \mathrm{mmHg}$ & 32 & 19.0 \\
\hline
\end{tabular}

$(p=0.1)$ and high preoperative IOP $(p=0.7)$ (Table 5).

\section{Discussion}

Combined Visco-Trab aims, in a single procedure, to lower IOP and lessen the early postoperative complications in patients with severe glaucoma. Surgeons familiar with trabeculectomy and nonpenetrating surgery will find no difficulty in performing Visco-Trab without any learning curve. In Visco-Trab, unlike viscocanalostomy, the technique is easier since dissection is not extending over Descemet's membrane where most macro-perforations occur. [5-9]. 
Fig. 2 Kaplan-Meier survival analysis of probability of failure of Visco-Trab to achieve a pressure of $14 \mathrm{mmHg}$ or less necessary for management of advanced glaucoma over the follow-up period

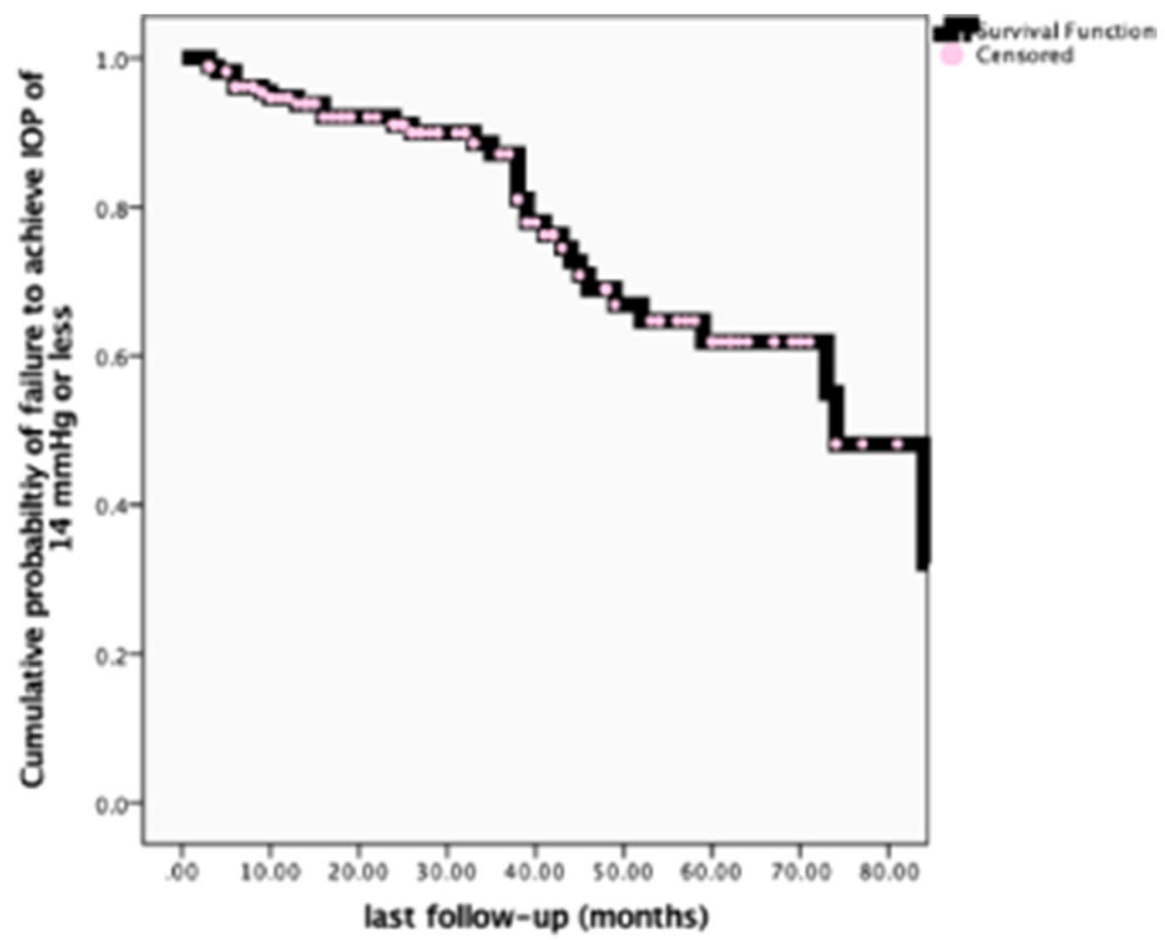

Table 5 Predictors of failure to achieve IOP of $14 \mathrm{mmHg}$ or less (logistic regression analysis)

\begin{tabular}{|c|c|c|c|c|c|}
\hline & \multirow[t]{2}{*}{ Regression coefficient } & \multirow[t]{2}{*}{$p$ value } & \multirow[t]{2}{*}{ Odd's ratio } & \multicolumn{2}{|c|}{$95 \%$ Confidence Interval } \\
\hline & & & & Lower & Upper \\
\hline Old age & 0.831 & 0.114 & 2.295 & 0.818 & 6.435 \\
\hline Preoperative IOP $(>25 \mathrm{mmHg})$ & 0.218 & 0.653 & 1.244 & 0.480 & 3.219 \\
\hline Number of preoperative glaucoma drops & 1.014 & $0.029 *$ & 2.757 & 1.108 & 8.860 \\
\hline Previous glaucoma operation & 1.089 & $0.040 *$ & 2.972 & 1.048 & 8.422 \\
\hline Previous ocular surgery & 1.380 & $0.011 *$ & 3.974 & 1.373 & 11.499 \\
\hline Severity of glaucoma & 0.936 & 0.058 & 2.550 & 0.970 & 6.702 \\
\hline
\end{tabular}

* indicate significant $p$ value

The combined Visco-Trab operation reduces IOP by enhancing both internal and external filtration of aqueous fluid. The internal filtration is enhanced by several mechanisms; (1) deep scleral flap dissection and excision creates a scleral lake that accommodates and directs aqueous to orifices of SC or to suprachoroidal space via the remaining thin scleral sheet; (2) dilation of SC with viscoelastic results in circumferential expansion of the canal and collector channels and focal ruptures of the inner wall of SC and juxta canalicular meshwork; (3) tight closure of the superficial lamellar scleral flap limits external flow to the subconjunctival space and directs all aqueous into conventional outflow pathways. On the other hand, subconjunctival flow is guaranteed over the long term via; (1) excision of a penetrating deep sclero-keratectomy (trabeculectomy) creates a guarded external fistula; (2) use of subconjunctival mitomycin $\mathrm{C}$ as an antifibrotic agent to reduce episcleral fibrosis; (3) delayed laser cutting of the sutures of the lamellar flap to augment external filtration when needed.

In this study, Visco-Trab operation for management of advanced glaucoma proved midterm efficacy in reducing IOP to $14 \mathrm{mmHg}$ or less in $60 \%$ (complete 
success) and $81 \%$ (qualified success) of the eyes at the last follow-up. The total success rate increased to $95.3 \%$ when a pressure of $21 \mathrm{mmHg}$ or less was used as a cutoff value. Our results were comparable to those of King and Stead [10]. They reported a success rate of $85 \%$ for achieving an IOP of $16 \mathrm{mmHg}$ or less at year 5 follow-up after MMC-Trab for patients with advanced glaucoma. However, Law and associates [11], comparing initial versus repeat MMC-Trab for open-angle glaucoma, reported lower success rate in achieving an IOP of $15 \mathrm{mmHg}$ or less $(61 \%$ and $41 \%$, respectively). On studying the outcome of viscocanalostomy in patients with advanced glaucoma at 1 , 2 , and 3 years follow-up by Tsagkataki and associates [12], complete success was $45 \%, 28 \%$, and $31 \%$, whereas qualified success was $67 \%, 66 \%$, and $60 \%$, respectively. On the other hand, combination of trabeculectomy with deep sclerectomy had an $83 \%$ complete success and $100 \%$ qualified success to achieve a $22 \mathrm{mmHg}$ or less IOP after 12 months of follow-up [13]. The success rate was much less in a recent study by Sangtam and associates [14] using combined modified deep sclerectomy and trabeculectomy for various types of glaucoma at different stages of severity. Complete success in their study was $50 \%$ (for IOP $<22 \mathrm{mmHg}$ ) and $36 \%$ (for IOP $<16$ $\mathrm{mmHg}$ ), while qualified success was $70 \%$ and $47 \%$, respectively. The difficulty to compare different studies in terms of success rates is due to variability in selection criteria of patient type, glaucoma diagnosis, disease severity; previous ocular surgery; primary versus repeat glaucoma operation; variable success criteria; and follow-up duration. In our Visco-Trab study, we had patients with various ethnicities, different types of glaucomas, and nearly one third of the eyes had previous ocular surgery, but all patients were categorized as advanced disease, operated by one surgeon, all had the same surgical technique, followup regimen, and criteria of assessment.

In the meantime, Visco-Trab operation safely reduced postoperative pressure spikes as well as hypotony-related complications that might result from excessive external filtration. Reported intraoperative complications were very few (Table 2) denoting that the procedure can be mastered easily. Micro- or macro-perforations are the most common complications of viscocanalostomy that occur during dissection of the descemet's window, a step not required in Visco-Trab operation. Postoperative complications that required added surgical intervention included flat anterior chamber ( 3 eyes), choroidal effusion ( 3 eyes, 2 of them had choroidal tap). None of the eyes lost light perception or had other devastating complications (endophthalmitis, or atrophia). In a previous study [3], early postoperative complications were comparable in number; however, some complications were greater in severity after MMC-Trab than after Visco-Trab, such as anterior chamber shallowness (no eyes with flat chamber in the Visco-Trab group compared to 5 eyes in the MMC-Trab group), choroidal effusion (none required surgical drainage after Visco-Trab compared to 2 eyes after MMCTrab), and surgically induced astigmatism. Additionally, patients required less postoperative surgical intervention in the Visco-Trab group (3 interventions compared to 8 in the MMC-Trab group). In another contralateral eye-controlled study [4], early postoperative complications were greater and postoperative surgical interventions were more frequent after MMCTrab than after Visco-Trab operation. In a previous study on 60 eyes with advanced glaucoma having MMC-Trab, a 35\% postoperative complication rate was reported (25\% of the eyes had excessive filtration and shallow or flat anterior chamber and $15 \%$ had choroidal effusion). [15] A similar rate of postoperative complications after Trab with antifibrotic agents was reported in other studies. [16-19].

Several studies reported post-laser suture lysis complication rates between 21 and $42 \%$ after MMCTrab [16, 20]. Viscoelastic dilatation of Schlemm's canal in Visco-Trab was shown to enhance internal filtration early postoperatively. This made suture cutting seldom necessary in the first two weeks after surgery despite tight closure of lamellar sclera flap. This relatively long interval may limit excessive flow of aqueous into the subconjunctival space with less adverse events. Morinelli and associates reported that a longer time interval between surgery and suture lysis may result in both a lesser degree of IOP reduction and a lower incidence of subsequent hypotony [20]. Delayed suture cutting postoperatively after ViscoTrab might be associated with early onset episcleral and scleral fibrosis. This, in some patients, may explain the lack of IOP lowering after laser suture lysis and necessitates invasive maneuvers such as needling and flap lift on the slitlamp (14 eyes) or bleb revision with subconjunctival mitomycin $\mathrm{C}$ in the operating room (11 eyes). 
Most of the eyes had stable visual acuity and visual fields compared to preoperative level (Table 3). None of the eyes had "wipe-out phenomenon" after ViscoTrab neither in this study nor in our previous report $[3,4]$. Costa and associates [21] reported that the risk of unexplained postoperative loss of central visual field does exist but is lower than $1 \%$ and is more likely to occur in older patients with macular splitting in the preoperative visual field. In another study, Topouzis et al. [22] reported no occurrences of "wipe-out" phenomenon in their series of end-stage glaucoma after MMC-Trab. They concluded that this sudden, unexplained postoperative loss of central vision is, at most, a rare complication and early surgical intervention should be considered in these patients. Stability of the visual filed mean deviation, cup/disc ratio, and visual acuity over the extended follow-up period (Table 3) indicates the importance of a low and stable target IOP for those glaucoma patients with advanced neuronal damage [23, 24].

Combined Visco-Trab, unlike nonpenetrating surgeries, can be performed for all types of glaucoma regardless of the angle appearance. Disadvantages of this technique include added surgical difficulty and time consumption in deep scleral block dissection \& Schlemm's canal exposure, extra cost of viscoelastic, a special knife and cannula not routinely used with MMC-Trab. In this study, sodium hyaluronate $1 \%$ is used instead of the high molecular weight Healon GV or Healon 5 which may have affected our results [25]. Tanito and coworkers [26] reported a comparable success rate using Healon 1\% (Pharmacia, Japan), but they assumed that it may be related to the high incidence of postoperative hyphema.

Two third of eyes had functioning blebs either diffuse (59 eyes, 35\%) or thin ischemic (54 eyes, 32\%) which may be matching with almost $60 \%$ of eyes that achieved complete success. On the other hand, only a quarter of the eyes $(45,26.8 \%)$ required postoperative laser suture lysis despite the surgical plan of tight closure of the lamellar scleral flap in Visco-Trab. This significant long-term IOP reduction without suture cutting may be attributed partly to viscoelastic dilation of Schlemm's canal and widening of trabecular pores which may provide long-lasting enhancement of internal filtration. In the meantime, even though the scleral flap is being closed watertight, there could be percolation of aqueous into the subconjunctival space assisted by blinking, ocular pulsation, digital massage, and reduced healing activity induced by antifibrotic agents (mitomycin $\mathrm{C}$ and topical steroids). Many of these patients develop a functioning bleb without the need for postoperative suture cutting.

Significant predictors for failure of Visco-Trab operation to reduce IOP to $14 \mathrm{mmHg}$ or less at last follow-up evaluation included previous ocular surgery $(p=0.01)$, previous glaucoma surgical intervention $(p=0.04)$ surgery, and number of preoperative antiglaucoma medications $(p=0.029)$. Severity of glaucoma (advanced versus end stage) had border line significance $(p=0.058)$, whereas old age $(p=0.1)$ and high preoperative IOP $(p=0.7)$ were not significant predictors for failure to maximally reduce IOP after Visco-Trab technique. Law and coworkers ${ }^{11}$ reported that younger age and requirement for laser suture lysis were significant risk factors for failures in eyes with repeated MMC-Trab. Song and associates [27] found increased age, greater baseline IOP, limbus-based conjunctival flaps, and MMC duration $>1$ min were associated with decreased risk of surgical failure of MMC-Trab for eyes with primary angle-closure glaucoma.

In conclusion, combined Visco-Trab operation proved safe and effective, on midterm follow-up, in reducing IOP to the proposed target level in eyes with severe glaucoma via enhancing internal and external filtration.

Author contributions First author contributed the main idea of the project, the surgical technique for all patients, manuscript writing. Second and third authors contributed clinical follow-up of the patients, data collection, data entry, and analysis.

Funding None.

Data availability All data available in data collection sheets and in SPSS files.

\section{Compliance with ethical standards}

Conflict of interest Tarek M. Eid Ezz, El-Din M. Ibrahim and Ahmad Zaid declares that he has no conflict of interest with any items related to this study.

Consent to participate All patients signed an informed consent.

Consent to publish Approved from authors.

Ethical approval All procedures performed in studies involving human participants were in accordance with the 
ethical standards of the institutional and/or national research committee and with the 1964 Helsinki Declaration and its later amendments or comparable ethical standards.

Informed consent Informed consent was obtained from all individual participants included in the study.

Open Access This article is licensed under a Creative Commons Attribution 4.0 International License, which permits use, sharing, adaptation, distribution and reproduction in any medium or format, as long as you give appropriate credit to the original author(s) and the source, provide a link to the Creative Commons licence, and indicate if changes were made. The images or other third party material in this article are included in the article's Creative Commons licence, unless indicated otherwise in a credit line to the material. If material is not included in the article's Creative Commons licence and your intended use is not permitted by statutory regulation or exceeds the permitted use, you will need to obtain permission directly from the copyright holder. To view a copy of this licence, visit http://creativecommons.org/licenses/by/4.0/.

\section{References}

1. Gessesse GW, Damji KF (2013) Advanced glaucoma: management pearls. Middle East Afr J Ophthalmol 20:131-141

2. Medeiros FA, Pinheiro A, Moura FC, Leal BC, Susanna R (2002) Intraocular pressure fluctuations in medical versus surgically treated glaucoma patients. J Ocul Pharmacol Ther 18:489-498

3. Eid TM (2008) Combined viscocanalostomy-trabeculectomy for management of far-advanced glaucoma. Evaluation of early postoperative course. Ophthalmic Surg Lasers Imaging 39:358-366

4. Eid TM, Tantawy W (2011) Combined viscocanalostomytrabeculectomy for management of advanced glaucoma. A comparative study of the contralateral eye. A pilot study. Middle East Afr J Ophthalmol (MEAJO) 18:292-297

5. Stegmann R, Piennar A, Miller D (1999) Viscocanalostomy for open-angle glaucoma in black African patients. J Cataract Refract Surg 25:316-322

6. Johnson DH, Johnson M (2001) How does nonpenetrating glaucoma surgery work? Aqueous outflow resistance and glaucoma surgery. J Glaucoma 10:55-67

7. Sanchez E, Schnyder CC, Mermoud A (1997) Comparative results of deep sclerectomy transformed to trabeculectomy and classical trabeculectomy. Klin Monatsbl Augenheilkd 210:261-264

8. Rebolleda G, Munoz-Negrete FJ (2005) Comparison between phaco-deep sclerectomy converted into phacotrabeculectomy and uneventful phaco-deep sclerectomy. Eur J Ophthalmol 15:343-346

9. Drusedau MU, Von Wolff K, Bull H et al (2000) Viscocanalostomy for primary open-angle glaucoma: the Gross Pankow experience. J Cataract Refract Surg 26:1367-1415
10. King AJ, Stead RE (2011) Outcome of trabeculectomy with mitomycin $\mathrm{C}$ in patients with advanced glaucoma. $\mathrm{Br} \mathrm{J}$ Ophthalmol 95:960-965

11. Law SK, Shih K, Tran DH, Coleman AL, Caprioli J (2009) Long-term outcomes of repeat versus initial trabeculectomy in open-angle glaucoma. Am J Ophthalmol 148:685-695

12. Tsagkataki M, Bampouras TMM, Chouldhary A (2018) Outcomes of viscocanalostomy and phaco-viscocanalostomy in patients with advanced glaucoma. Graefe's Arch ClinExp Ophthalmol 256:1481-1487

13. Kayikcioglu OR, Emre S, Kaya Z (2010) Trabeculectomy combined with deep sclerectomy and scleral flap suture tension adjustment under an anterior chamber maintainer: a new modification of trabeculectomy. Int Ophthalmol 30:271-277

14. Sangtam T, Roy S, Mermoud A (2020) Outcome and complications of combined modified deep sclerectomy and trabeculectomy for surgical management of glaucoma. Clin Ophthalmol 14:795-803

15. Eid TM (2005) Clinical outcome of trabeculectomy with adjunctive mitomycin in patients with advanced glaucoma and poor compliance to medical assessment. Bull Ophthalmol Soc Egypt 98:395-400

16. Blindish R, Condon GP, Schosser JD et al (2002) Efficacy and safety of mitomycin $\mathrm{C}$ in primary trabeculectomy: fiveyear follow-up. Ophthalmology 109:1336-1341

17. Anand N, Meilke C, Daeda VK (2001) Trabeculectomy outcomes in advanced glaucoma in Nigeria. Eye 15:274-278

18. The Fluorouracil Filtering Surgery Study Group (1996) Five-year follow-up of the fluorouracil filtering surgery study. Am J Ophthalmol 121:349-366

19. Zacharaia PT, Deppermann SR, Schuman JS (1993) Ocular hypotony after trabeculectomy with mitomycin C. Am J Ophthalmol 116:314-326

20. Morinelli EN, Sidoti PA, Heuer DK et al (1996) Laser suture lysis after mitomycin $\mathrm{C}$ trabeculectomy. Ophthalmology 103:306-314

21. Costa VP, Smith M, Spaeth GL, Gandham S, Markovitz B (1993) Loss of visual acuity after trabeculectomy. Ophthalmology 100:599-612

22. Topouzis F, Tranos P, Koskosas A et al (2005) Risk of sudden visual loss following filtration surgery in end-stage glaucoma. Am J Ophthalmol 140:661-666

23. The Advanced Glaucoma Intervention Study (AGIS) 7 (2000) The relationship between control of intraocular pressure and visual field deteriorations. AGIS Invest Am J Ophthalmol 130:429-440

24. Leske MC, Heijl A, Hyman L, Bengtsson B, Dong L, Yang Z; EMGT Group (2007) Predictors of long-term progression in the early manifest glaucoma trial. Ophthalmology 114(11):1965-1972

25. Smit BA, Johnstone MA (2002) Effects of viscoelastic injection into Schlemm's canal in primate and human eyes. Potential Relevance Viscocanalostomy Ophthalmol 109:786-792

26. Tanito M, Park M, Nishikawa M, Ohira A, Chihara E (2002) Comparison of surgical outcomes of combined viscocanalostomy and cataract surgery with combined trabeculectomy and cataract surgery. Am J Ophthalmol 134:513-520 
27. Song BJ, Ramannathan M, Morales E et al (2016) Trabeculectomy and combined phacoemulsification-trabeculectomy: outcomes and risk factors for failure in primary angle closure glaucoma. J Glaucoma 25:763-769
Publisher's Note Springer Nature remains neutral with regard to jurisdictional claims in published maps and institutional affiliations. 